\title{
Resonant activation in a simple kinetic model
}

\author{
J.J. Brey and J. Casado-Pascual \\ Física Teórica, Universidad de Sevilla, Apartado Correos 1065, 41080 Sevilla, Spain
}

(Received 1 February 1994)

\begin{abstract}
We present a very simple Markovian kinetic model displaying a stochastic resonant behavior which is similar to the one found in the escape of a particle over a fluctuating potential barrier. The basic mechanism that is responsible for the existence of resonance is identified. This allows the generalization of the model in different ways, leading to a variety of models where a similar phenomenon is to be expected. It is also shown that the initial conditions play an important role in determining whether the resonant activation actually shows up.
\end{abstract}

PACS number(s): 05.40. $+\mathrm{j}, 82.20 . \mathrm{Mj}$

\section{INTRODUCTION}

In the past years, much attention has been devoted to the study of resonance effects in stochastic systems. The most extensively considered phenomenon is the noiseinduced enhancement of a small systematic periodic signal in nonlinear systems and it is usually referred to as stochastic resonance $[1,2]$. Recently, Doering and Gadona [3] reported the existence of another resonance effect in the escape rate over a linear barrier whose slope fluctuates between two values. The mean first passage time (MFPT) as a function of the flipping rate of the barrier presents a minimum, which was characterized as a resonant activation over the barrier.

In order to understand the origin of the resonant activation, it seems important to determine whether a similar phenomenon can be observed in simple kinetic models. Bier and Astumian [4] have analyzed a Markovian kinetic model in which the reactant switches at a given rate between two internal states. These two states have different rates of decay towards the final absorbing product state. For small flipping rates, the model is closely related to the escape over a linear fluctuating barrier in the limit of large barrier and small fluctuations. Nevertheless, the behavior of both systems for large flipping rates is quite different and the model does not exhibit resonant activation. On the other hand, Van den Broeck [5] had previously shown that the phenomenon can be displayed for non-Markovian variants of the same model. More concretely, he has considered the case of nonexponential waiting time distributions for the internal states of the reactant.

The purpose of this paper is to present a simple kinetic model showing, under certain circumstances, a resonant behavior that is similar to that reported in [3]. There are several reasons that render the relevance of the study of this model. First, it is Markovian, unlike those which have been considered previously [5]. Besides, its technical simplicity allows us to determine in a precise way the conditions under which the resonance effect arises and, in particular, the important role played by the initial conditions. Finally, the model clearly identifies the mechanism which is responsible for the resonance. It is due to the fluctuations of an intermediate state connecting the ini- tial and final states. These fluctuations must have opposite effects on the transitions from the intermediate state to the final and to the initial states. From this point of view, our model is a minimal model in the sense that it can be generalized in many different ways preserving the resonant behavior. In particular, it allows us to explain the behavior of the linear fluctuating barrier analyzed in [3].

The rest of this paper is organized as follows. In the next section, we describe our model and find exact results for the MFPT from the initial state (reactant) to the final state (product). The conditions under which resonant activation appears are investigated. In Sec. III we discuss some modified versions of the model that do not lead to resonant behavior. Comparison of the several models clearly shows the origin of stochastic resonance in this kind of models. We conclude with a brief summary of our main results.

\section{MODEL}

The model is described by the kinetic scheme depicted in Fig. 1. The transformation of the reactant $A$ into the product $C$ takes place through an intermediate substance $B$. This one, due, for instance, to the influence of some external conditions, randomly switches at a rate $\gamma$ between two states, denoted by $B^{+}$and $B^{-}$, respectively. The substance $B$ remains in one state for an exponentially distributed random time before switching to another and $\gamma$ is the inverse of the average time that $B$ stays in one state before switching. When $B$ is in state $B^{+}$, only transitions from $B$ to $A$ at rate $k_{1}$ are possible, while for $B$ being in state $B^{-}$the possible transitions

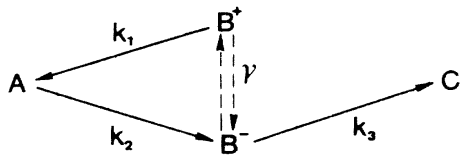

FIG. 1. Sketch of the kinetic model. The state of the intermediate substance $B$ fluctuates between $B_{+}$and $B_{-}$at rate $\gamma$. 
are from $A$ to $B$, at rate $k_{2}$, and from $B$ to $C$, at rate $k_{3}$. Thus the state of $B$ acts as a control variable for the transformation of $A$ into $C$.

Our aim is to find the noise-averaged evolution of the concentrations of $A$ and $B$. This is a typical problem of the so-called dynamical disorder and a general scheme for treating such problems has been reviewed by Zwanzig [6]. We introduce partially averaged concentrations of $\boldsymbol{A}$ and $B$ for a given state of $B$. They will be denoted by $P_{ \pm}(A, t)$ and $P_{ \pm}(B, t)$, where the signs + and - refer to states $B^{+}$and $B^{-}$, respectively. Then, for instance, $P_{+}(A, t)$ is the concentration of $A$ at time $t$, with $B^{+}$ the state of $B$. The actual concentration of $A$ at time $t$, $P(A, t)$, is given by

$$
P(A, t)=P_{+}(A, t)+P_{-}(A, t),
$$

and similarly for the concentration of $B, P(B, t)$. The partially averaged concentrations satisfy the equations

$$
\begin{gathered}
\frac{\partial}{\partial t} P_{+}(A, t)=-\gamma P_{+}(A, t)+k_{1} P_{+}(B, t)+\gamma P_{-}(A, t) \\
\frac{\partial}{\partial t} P_{-}(A, t)=-\left(k_{2}+\gamma\right) P_{-}(A, t)+\gamma P_{+}(A, t),
\end{gathered}
$$

$$
\begin{gathered}
\frac{\partial}{\partial t} P_{+}(B, t)=-\left(k_{1}+\gamma\right) P_{+}(B, t)+\gamma P_{-}(B, t), \\
\frac{\partial}{\partial t} P_{-}(B, t)=-\left(k_{3}+\gamma\right) P_{-}(B, t) \\
+k_{2} P_{-}(A, t)+\gamma P_{+}(B, t) .
\end{gathered}
$$

The initial condition is that only reactant $A$ is present, but nothing will be assumed about the initial distribution of states $B^{+}$and $B^{-}$. Therefore, we take

$$
\begin{gathered}
P_{+}(A, 0)=a, \quad P_{-}(A, 0)=1-a, \\
P_{+}(B, 0)=P_{-}(B, 0)=0,
\end{gathered}
$$

with $0 \leq a \leq 1$. The MFPT for the transformation of $A$ into $C$ is given by [7]

$$
\langle\tau\rangle=\int_{0}^{\infty} d t[P(A, t)+P(B, t)]
$$

and a simple calculation using, for instance, the Laplace transformed of Eq. (2) yields

$$
\langle\tau\rangle=\frac{2 \gamma^{2}\left(k_{1}+k_{2}+k_{3}\right)+2 \gamma k_{1}\left(k_{2}+k_{3}\right)+a \gamma k_{2} k_{3}+a k_{1} k_{2} k_{3}}{\gamma^{2} k_{2} k_{3}+\gamma k_{1} k_{2} k_{3}} .
$$

The behavior of $\langle\tau\rangle$ in the two limiting values of $\gamma$ is easily understood. When $\gamma$ is very large, the above result becomes

$$
\langle\tau\rangle \rightarrow\langle\tau\rangle_{\infty}=\frac{2\left(k_{1}+k_{2}+k_{3}\right)}{k_{2} k_{3}},
$$

which does not depend on the value of the initial condition $a$. In fact, this is the expression one gets by computing the MFPT for the process

$$
A \rightleftharpoons B \rightarrow C
$$

and substituting the transition rates by their equilibrium values, which are $k_{1} / 2$ for the transition from $B$ to $A$, $k_{2} / 2$ for the transition from $A$ to $B$, and $k_{3} / 2$ for the transition from $B$ to $C$.

On the other hand, in the static disorder limit $\gamma \rightarrow 0$, $\langle\tau\rangle$ goes to infinity (except in the particular case $a=0$ ) because the transition from $A$ to $C$ is not possible when $B$ is in the state $B^{+}$. The question now is whether the MFPT presents a minimum between the above two limiting values. From Eq. (6) it follows that such a minimum exists if

$$
2 k_{1}^{2}>a k_{2} k_{3} .
$$

Moreover, the minimum is attained for a switching rate

$$
\gamma_{0}=\frac{k_{1}\left(a k_{2} k_{3}\right)^{1 / 2}}{2^{1 / 2} k_{1}-\left(a k_{2} k_{3}\right)^{1 / 2}}
$$

and it is given by

$$
\langle\tau\rangle_{0}=-\frac{a}{k_{1}}+\frac{2}{k_{2}}+\frac{2}{k_{3}}+\frac{2^{3 / 2} a^{1 / 2}}{\left(k_{2} k_{3}\right)^{1 / 2}} .
$$

Therefore, a resonance effect occurs when the initial probability distribution of the fluctuating states of $B$, characterized by the parameter $a$, and the rates $k_{1}, k_{2}$, and $k_{3}$ verify the relation given in Eq. (9). The relevant role played by the initial condition to determine whether there is resonance must be noticed. In particular, for given values of the transition rates, there will always be resonance for small enough values of $a$. As an example, we have plotted in Fig. 2 the MFPT as a function of $\gamma$ for $k_{1}=k_{2}=k_{3}=1$. The initial condition is $a=1 / 2$, which

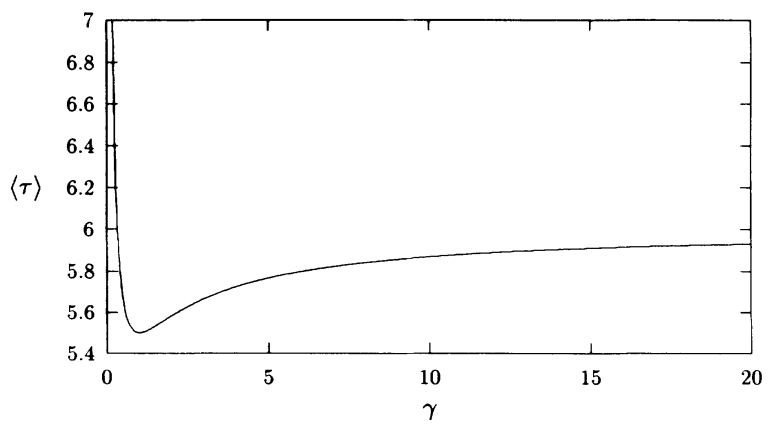

FIG. 2. Mean first passage time as a function of the switching rate for the model sketched in Fig. 1. The values of the parameters are $k_{1}=k_{2}=k_{3}=1$ and the initial condition is $a=1 / 2$. 
corresponds to the steady distribution of the switching mechanism if it were isolated. As predicted by Eq. (10), the resonance takes place for $\gamma_{0}=1$.

The origin of the resonance shows up clearly if we rewrite Eq. (6) as

$$
\langle\tau\rangle=\frac{a}{\gamma}+\frac{2 k_{1}}{k_{2} k_{3}} \frac{\gamma}{\gamma+k_{1}}+2\left(\frac{1}{k_{2}}+\frac{1}{k_{3}}\right) .
$$

The flipping rate appears in two summands on the right hand side of this expression. The first one is a decreasing function of $\gamma$ and reduces to zero for $\gamma \rightarrow \infty$ or $a=$ 0 , while the second one is an increasing function of $\gamma$ and tends to the asymptotic value $2 k_{1} / k_{2} k_{3}$. Besides, the latter does not depend on the initial condition $a$. Therefore, the flipping plays a twofold role. On the one hand, it facilitates the decay of the initial probability of finding the system in the configuration in which the transition to the product $C$ is impossible. On the other hand, it makes it that not all the transitions from $A$ to $B$ proceed up to $C$. Some of the substance reaching $B$ is returned to $A$. The competition of these two roles leads in some cases to resonant activation. The condition is that the second tendency dominates for large enough flipping rates.

If we introduce a measure $R$ of the resonance effect as

$$
R=\langle\tau\rangle_{\infty}-\langle\tau\rangle_{0}
$$

it is seen from Eqs. (7) and (11) that, for a given value of $a$, the resonance is stronger the larger the value of $k_{1}$ and the smaller the values of $k_{2}$ and $k_{3}$. In the limit $k_{1} \rightarrow \infty$,

$$
\gamma_{0} \rightarrow \frac{\left(a k_{2} k_{3}\right)^{1 / 2}}{2^{1 / 2}}
$$

and $R$ diverges as a consequence of the divergence of $\langle\tau\rangle_{\infty}$

\section{VARIANTS AND GENERALIZATIONS OF THE MODEL}

In order to identify the basic mechanism that is responsible for the resonance behavior, we now turn to the

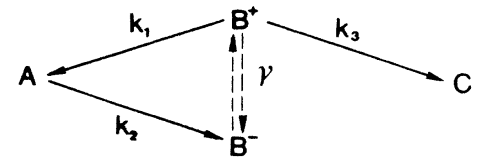

(a)

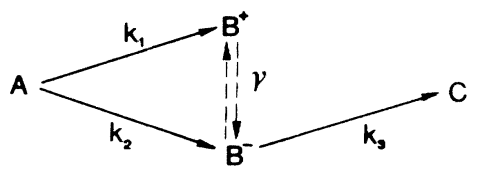

(b)

FIG. 3. Two variants of the kinetic model discussed in Sec. II.

variant of the model that is sketched in Fig. 3(a). The interpretation of the scheme is similar to the one in Fig. 1 and the only difference with the previous model is that now the decay to the product $C$ occurs when the intermediate substance $B$ is in state $B^{+}$. Using the same procedure as above and the initial conditions given by Eqs. (3) and (4), the MFPT for this model is obtained as

$$
\langle\tau\rangle=\frac{2 \gamma\left(k_{1}+k_{2}+k_{3}\right)+2 k_{1} k_{2}+(1+a) k_{2} k_{3}}{\gamma k_{2} k_{3}} .
$$

In the limit $\gamma \rightarrow \infty$, this expression reduces to Eq. (7). Thus, both models have the same behavior in the high frequency limit. This is the expected result since the discussion given below Eq. (7) also applies in this case. Nevertheless, from Eq. (15) one gets

$$
\frac{\partial\langle\tau\rangle}{\partial \gamma}=-\frac{2 k_{1}+(a+1) k_{3}}{\gamma^{2} k_{3}}
$$

which indicates that the MFPT is a monotonously decreasing function of $\gamma$ for all values of $k_{1}, k_{2}, k_{3}$, and $a$ and therefore this model does not present the resonance phenomenon.

Let us still consider another variant, namely, that described by Fig. 3(b). The expression of the MFPT for it reads

$$
\langle\tau\rangle=\frac{2 \gamma^{2}\left(k_{1}+k_{2}+k_{3}\right)+\gamma\left(2 k_{1} k_{2}+(2-a) k_{1} k_{3}+a k_{2} k_{3}\right)+a k_{1} k_{2} k_{3}}{\gamma^{2} k_{3}\left(k_{1}+k_{2}\right)+\gamma k_{1} k_{2} k_{3}} .
$$

Taking into account that $0 \leq a \leq 1$, it is easily seen that we again have a monotonous decay. Let us point out that the high frequency limit for this model is

$$
\langle\tau\rangle_{\infty}=\frac{2\left(k_{1}+k_{2}+k_{3}\right)}{k_{3}\left(k_{1}+k_{2}\right)}
$$

which is different from the result obtained in the same limit for the two previous models, Eq. (7).

What is the conclusion emerging from the compari- son of the three models we have analyzed? Consider the general model with a fluctuating intermediate state represented in Fig. 4. For $k_{1}, k_{2}$, and $k_{3}$ different from zero, the MFPT is a continuous function of all the transition rates. Therefore, our results suggest that the possibility of getting a resonant behavior requires that $k_{1}>k_{2}^{\prime}$, $k_{2}>k_{1}^{\prime}$, and $k_{3}>k_{4}$. The fluctuations of $B$ must be such that when the transition rate from $B$ to $C$ increases, the transition rate from $A$ to $B$ also increases, while that associated with the transformation from $B$ to $A$ becomes 


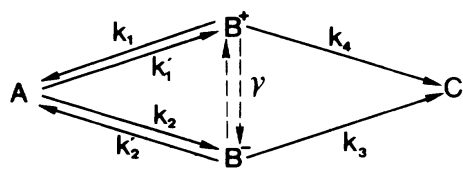

FIG. 4. Sketch of the general kinetic model with an intermediate fluctuating state.

smaller.

As an example, let us assunie that the process of going from $A$ to $C$ corresponds to the diffusion of a particle on a one dimensional lattice from the site $n=0$ to the site $n=2$, in the presence of an external potential field. The probability of jumping per unit of time is $\omega(1-\lambda)$ for jumps to the right and $\omega(1+\lambda)$ for jumps to the left, with $0<\lambda<1$. The parameter $\lambda$ is proportional to the slope of the external potential [8] and $w$ is a natural frequency of the lattice, which will be used to fix the time scale and therefore it will be taken equal to unity in the following.

Now, suppose that $\lambda$ fluctuates, with a flipping rate $\gamma$, between two values $\lambda_{+}$and $\lambda_{-}$as a consequence of the fluctuation of the slope of the external potential. The partially averaged probabilities of finding the particle at site $n$, for a given value of $\lambda$, obey the master equation

$$
\begin{aligned}
\frac{\partial}{\partial t} P_{+}(1, t)= & -\left(1-\lambda_{+}+\gamma\right) P_{+}(1, t) \\
& +\left(1+\lambda_{+}\right) P_{+}(2, t)+\gamma P_{-}(1, t) \\
\frac{\partial}{\partial t} P_{-}(1, t)= & -\left(1-\lambda_{-}+\gamma\right) P_{-}(1, t) \\
& +\left(1+\lambda_{-}\right) P_{-}(2, t)+\gamma P_{+}(1, t) \\
\frac{\partial}{\partial t} P_{+}(2, t)= & -(2+\gamma) P_{+}(2, t) \\
& +\left(1-\lambda_{+}\right) P_{+}(1, t)+\gamma P_{-}(2, t) \\
\frac{\partial}{\partial t} P_{-}(2, t)= & -(2+\gamma) P_{-}(2, t) \\
& +\left(1-\lambda_{-}\right) P_{-}(1, t)+\gamma P_{+}(2, t),
\end{aligned}
$$

where we have introduced a reflecting boundary at $n=0$ and an absorbing one at $n=2$. Since the model verifies the conditions formulated above, it is expected to present resonant activation in some region of its space of parameters. As for the other models, it is straightforward to obtain the MFPT using the initial conditions $P_{+}(1,0)=$ $a, P_{-}(1,0)=1-a$, and $P_{+}(2,0)=P_{-}(2,0)=0$. Nevertheless, the expression is very large and will be omitted here. In the limit $\gamma \rightarrow \infty$, it reduces to

$$
\langle\tau\rangle_{\infty}=\frac{2\left(6-\lambda_{+}-\lambda_{-}\right)}{\left(\lambda_{+}+\lambda_{-}-2\right)^{2}} .
$$

The existence of resonance can be analyzed by studying the first order correction in $\gamma^{-1}$ to $\langle\tau\rangle_{\infty}$. When this correction is negative, $\langle\tau\rangle$ must present a mininuun for some finite value of $\gamma$. For $a=1 / 2$, the system always exhibits resonant activation, independent of the values of $\lambda_{+}$and $\lambda_{-}$. Nevertheless, this is not true for other values of $a$. For instance, for $a=0.8, \lambda_{+}=0.2$, and $\lambda_{-}=0.1$, $\langle\tau\rangle$ decays monotonously to $\langle\tau\rangle_{\infty}$.

If the number of intermediate states $B$ is increased, the transition probabilities are scaled with the density of sites, and the continuous limit is taken, the system reduces [8] to the Brownian motion in a fluctuating linear barrier studied by Doering and Gadona [3]. Let us remark that their calculations correspond to the initial condition $a=1 / 2$ and therefore it is not surprising that they found resonance for all pairs of values of the slope of the barrier. In view of the analysis presented here, we speculate that the result could be different for other initial conditions.

\section{SUMMARY AND CONCLUSIONS}

In this paper we have shown that the resonance phenomenon referred to as resonant activation can be understood in terms of a simple kinetic model. The main feature of the model is the presence of an intermediate fluctuating state. The technical simplicity of the master equation governing the time evolution of the system allows us to obtain an explicit exact expression for the mean first-passage time in terms of the parameters defining the model and the initial conditions. In this way, a detailed analysis of the conditions required for the display of resonant behavior has been possible. A necessary condition seems to be that the fluctuations of the intermediate state have different qualitative effects on the transition rates corresponding to processes pointing in the direction of the final state and on those associated with processes in the opposite direction.

An interesting feature shown here is the effect of the initial conditions on the resonance. They are not only influencing the amplitude of the phenomenon, but actually determining whether it will take place or not. This is something to be taken into account upon designing experiments looking for resonant activation. In this context, although we have not tried to relate our model to any real system, it is clear from its structure that it can be useful for the description of some chemical transitions. Besides, generalizations of the model, like the one described in Sec. III, can be applied to a variety of physical problems.

\section{ACKNOWLEDGMENTS}

Partial support from the Dirección General de Investigación Científica y Técnica (Spain) through Grant No. PB92-0683 is gratefully acknowledged. 
[1] B. McNamara and W. Wiesenfeld, Phys. Rev. A 39, 4854 (1989).

[2] A. Bulsara and F. Moss, J. Stat. Phys. Special Issues $(1,2)$ 70 (1993).

[3] C. R. Doering and J. C. Gadona, Phys. Rev. Lett. 69, 2318 (1992); U. Zürcher and C. R. Doering, Phys. Rev. E 47, 3862 (1993).

[4] M. Bier and R. D. Astumian, Phys. Rev. Lett. 71, 1649 (1993).
[5] C. Van den Broeck, Phys. Rev. E 47, 4579 (1993).

[6] R. Zwanzig, Acc. Chem. Res. 23, 148 (1990). Processes of the same kind are treated in Ref. [7], Sec. VII.7, where they are called "composite Markov processes."

[7] N. G. van Kampen, Stochastic Processes in Physics and Chemistry (North-Holland, Amsterdam, 1992).

[8] See, for instance, Stochastic Processes in Physics and Chemistry (Ref. [7]), Sec. XI.2. 